\title{
Acknowledgment to Reviewers of Neurology International in 2021
}

\author{
Neurology International Editorial Office
}

Citation: Neurology International

Editorial Office. Acknowledgment to

Reviewers of Neurology International

in 2021. Neurol. Int. 2022, 14,

174-175. https://doi.org/10.3390/

neurolint14010013

Published: 25 January 2022

Publisher's Note: MDPI stays neutral with regard to jurisdictional claims in published maps and institutional affiliations.

Copyright: $\odot 2022$ by the author. Licensee MDPI, Basel, Switzerland.

This article is an open access article distributed under the terms and conditions of the Creative Commons Attribution (CC BY) license (https://creativecommons.org/licenses/by/4.0/).

MDPI AG, St. Alban-Anlage 66, 4052 Basel, Switzerland

Rigorous peer-reviews are the basis of high-quality academic publishing. Thanks to the great efforts of our reviewers, Neurology International was able to maintain its standards for the high quality of its published papers. Thanks to the contribution of our reviewers, in 2021, the median time to first decision was 29 days and the median time to publication was 57 days. The editors would like to extend their gratitude and recognition to the following reviewers for their precious time and dedication, regardless of whether the papers they reviewed were finally published:

Aaron Lerner

Abeer Al-Gharaibeh

Adam Fleming

Adina Turcu-Stiolica

Ah-Mee Park

Ajinkya S. Sase

Alberto Verrotti

Albrecht Gunther

Alexander Weil

Alexandre González-Rodríguez

Alexey Boyko

Amad Zafar

Amy McTague

André Toulouse

Andrea Alexandre

Andrea Bartoli

Andrew C. Gallup

Anna Jamroz-Wiśniewska

Axel Steiger

Ayataka Fujimoto

Barbara Remberk

Bartłomiej Henryk Noszczyk

Bernard Possidente

Briana R. De Miranda

Chenjie Shen

Chun-An Cheng

Cristina Daia

Cyrus Motamed

Daniela Calina

Danuta Szkutnik-Fiedler

Dario Siniscalco

Daša Stupica
David Kachlík

Davide Luglietto

Diego Cotella

Dragos Catalin Jianu

Duncan Hill

Elena Savvateeva

Elisa Maseroli

Emanuel Vamanu

Emmanuel Cognat

Enrico Opocher

Esteban Sepúlveda

Farid Rahimi

Federico Paolini Paoletti

Filippo M. Santorelli

Francesco Asci

Francesco Corea

Frédéric Gilbert

Gaëtan Lesca

Georgios Tsivgoulis

Gerrit Brinker

Ghulam Md Ashraf

Gina Ferrazzano

Giorgio Callovini

Giovanni Ferrara

Gonçalo S. Duarte

Goran Krstacic

Hans-Peter Hartung

Henry H. Woo

Hongsun Guo

Howan Leung

Ilona Rubi-Fessen

Jakov Milić 
Jan Purrucker

Johann Sellner

John Matsoukas

Juan Carlos López-García

Judith Drenthen

Kabirullah Lutfy

Kally C. O'Reilly

Katarzyna Hojan

Kathrine Bach Søndergaard

Kazuhiro P. Izawa

Keun-Yeong Jeong

Kunal Dhiman

Kurt Svardsudd

Lawrence S. Honig

Liana Dehelean

Luigi F. Iannone

Mahesh Kaushik

Marcello Moccia

Marcin Arciszewski

Marek Krzystanek

Margot Ernst

Maria Giuseppina Miano

Marsha Pierce

Martin Regensburger

Ming Chen

Mittal Jasoliya

Monokesh Kumer Sen

Muck-Seler Dorotea

Mujeeb U. Shad

Muneaki Matsuo

Natalia Madetko
Nathan K. Evanson

Neepa J. Patel

Nicola Montemurro

Nikolaos Pitsikas

Noriaki Tomura

Pamela McCombe

Paolo Immovilli

Patrycja Kleczkowska

Pierre Eric Juif

Raquel Barbosa

Reem Masarwa

Renata Balnyte

riantafyllos Didangelos

Rita Maria Vittoria Nobili

Roald Flesland Havre

Rogério Leone Buchaim

Roman Trochimczuk

Romina Romaniello

Rosa De Micco

Rubén Darío Castro-Torres

Samuel X. Shi

Sergio Ferrari

Shamik Shah

Shitiz Sriwastava

Srihari Gopal

Steffen Grautoff

Taku Sugiyama

Thorsten Rudroff

Tomasz Frączyk

Tomohiko Sadaoka

Vaitsa Giannouli 